\title{
Reply to Astarita et al. Comment on “Celentano et al. Suitability of a Progenitor Cell-Enriching Device for In Vitro Applications. Coatings 2021, 11, 146"
}

\author{
Antonio Celentano ${ }^{1, *(\mathbb{D}}$, Tami Yap ${ }^{1}\left(\mathbb{D}\right.$, Giuseppe Pantaleo ${ }^{2}$, Rita Paolini ${ }^{1}$, Michael McCullough ${ }^{1}$ and \\ Nicola Cirillo ${ }^{1}$ \\ 1 Melbourne Dental School, The University of Melbourne, 720 Swanston Street, Carton, VIC 3053, Australia; \\ tspyap@unimelb.edu.au (T.Y.); rita.paolini@unimelb.edu.au (R.P.); m.mccullough@unimelb.edu.au (M.M.); \\ nicola.cirillo@unimelb.edu.au (N.C.) \\ 2 Department of Medicine and Surgery, University of Salerno, Via Salvador Allende, 43, \\ 84081 Baronissi, Salerno, Italy; gpantaleo@unisa.it \\ * Correspondence: antonio.celentano@unimelb.edu.au; Tel.: +61-3-9341-1495
}

check for updates

Citation: Celentano, A.; Yap, T.; Pantaleo, G.; Paolini, R.; McCullough, M.; Cirillo, N. Reply to Astarita et al. Comment on "Celentano et al. Suitability of a Progenitor CellEnriching Device for In Vitro Applications. Coatings 2021, 11, 146". Coatings 2021, 11, 741. https:// doi.org/10.3390/coatings11070741

Academic Editor: Roberto Lo Giudice

Received: 9 June 2021

Accepted: 16 June 2021

Published: 22 June 2021

Publisher's Note: MDPI stays neutral with regard to jurisdictional claims in published maps and institutional affiliations.

Copyright: (c) 2021 by the authors. Licensee MDPI, Basel, Switzerland. This article is an open access article distributed under the terms and conditions of the Creative Commons Attribution (CC BY) license (https:/ / creativecommons.org/licenses/by/ $4.0 /)$.
We would like to thank HBW representatives for their comments [1] regarding our recently published article [2]. We understand that some of the concerns they have raised could have been generated as a result of misinterpretation of our article.

In particular, upon reading our article, Astarita and colleagues did not seem to understand that we did not aim to evaluate, judge, or critically appraise the clinical performance of this well-established Class 2a medical device. We made no statements to discourage the use of the device clinically; conversely, we reported that the usefulness and clinical validity of the Rigenera protocol has been widely documented with robust literature to date in several fields of regenerative medicine, as clearly depicted by these authors in the Introduction section: "At present, Rigenera ${ }^{\circledR}$ is an innovative and promising application in regenerative medicine, particularly in the fields of dental surgery, dermatology, plastic surgery, maxillafacial surgery, orthopaedics, aesthetic medicine, and wound healing [7-15]." The overall tone of our Introduction section was clearly optimistic about the clinical applications of the device, and the great majority of the respective references were provided to the readers for their perusal.

Conversely, the sole aim of our study was to test a new/off-label use of the device to assess the potential value of this technology for in vitro applications/laboratory use. Unfortunately, as clearly described in the third paragraph of our discussion, "in our experimental conditions", and as often happens during the early phases of new studies, the results obtained were not suggestive of any further space for this device in an in vitro context. Our results sharply and honestly reported what was found in our experimental conditions, and being highly reproducible, are of value for any scientist who would like to search for new protocols to be applied to in vitro settings.

The rationale that inspired our study from inception was only the ability of the device to be used safely in vitro in a laboratory environment, and eventually its ability to produce progenitor cells enriched samples based on separation by cell size selection. As often happens with new drugs and devices, we did not reach the second phase of our study because the results of the pilot tests were not satisfactory in our experimental settings.

We are happy to address point-by-point all concerns raised by HBW representatives.

- $\quad$ Regarding the first comment:

The aim of the paper has been clearly reported in only two sentences throughout the text, namely the Abstract section:

- "This study aimed to evaluate its potential use for in vitro cell models" and at the end of the Introduction section: 
- $\quad$ "we aimed to assess if the Rigenera ${ }^{\circledR}$ workflow could be utilized as an alternative cell sorting technique for laboratory research and further, to characterize the effect of Rigenera ${ }^{\circledR}$ workflow on the viability of primary cultured fibroblasts and on the quality of the processed samples."

Therefore, we were interested in processing, as a pilot study, a pool of primary fibroblasts (therefore potentially containing progenitor cells), to explore, for the first time, if an initial simple mechanically-driven size-selection process could produce, in vitro, a cluster of cells with different characteristics. We decided not to characterize the obtained samples further, given the results we obtained.

- Regarding the second comment:

The company insists that the device was "successfully" used in vitro in 16 studies, however, none of the cited studies systematically assessed, in vitro, the biocompatibility of the device, and the composition of the micrograft to discriminate for the presence of debris. On a side note, a few studies included in Table 1 were in fact not even pertinent. As an example, Xie et al. (2020) used the Rigenera protocol in vivo in a mouse myocardial infarction model. [3] Whereas, Jimi et al. (2017) investigated the mechanisms of skin wound healing by autologous micrograft in mice [4]. It is worth noting that several of these articles were co-authored by HBW members.

We would like to stress that we were indeed able to obtain viable cells using the device, however this does not mean that it can be used safely or recommended for in vitro research. In particular, the remarkable amount of large metal debris found in samples and controls warrants further development of the technique for optimal in vitro use.

- $\quad$ Regarding the third comment:

Once again, we believe that the raised concerns are mainly due to a misperception of our study. We carefully reported all the salient characteristics of the device, describing its components and the working principle of the device in detail, including clarification of the use of saline solution. Particularly, this is demonstrated in the fifth paragraph of the Introduction section, here reported for your perusal:

"Rigenera ${ }^{\circledR}$ consists of two main components: The Rigenera ${ }^{\circledR}$ machine, and Rigeneracons ${ }^{\circledR}$ (also known as Medicons ${ }^{\circledR}$ ). The Rigeneracons ${ }^{\circledR}$ are disposable devices activated by the machine that includes a metal steel grid and a blade. The grid is comprised of 100 hexagonal pores with indicated diameter sizes of 25, 50 and $75 \mu \mathrm{m}$. The blade is helical in shape and disaggregates the tissue samples placed in the Rigeneracons ${ }^{\circledR}$ creating mechanical friction against the grid at rotational speeds of $80 \mathrm{RPM}$, in the presence of saline solution [7]. Disaggregated single cells are mechanically guided through the pores in the grid, precipitating in a lower chamber from which they are collected with a sterile syringe for clinical use. Separation by cell size selection achieved with the Rigenera ${ }^{\circledR}$ system is the hallmark of the device. Stem cells are known to range from 20-40 $\mu \mathrm{m}$ in diameter [16], hence the processed micro-grafts are believed to contain a high population of progenitor cells, thus leading to optimal regenerative potential [12-17]. The processing times of the micro-grafts range from $10 \mathrm{~s}$ to minutes [7]."

Once describing our procedures, we always used terms such as "when applied in vitro", "for its potential in vitro use", "In our experimental conditions", referring the reader towards the $M \& M$ section where the experimental conditions have been clearly presented in order to be easily reproduced by anyone. We also want to point out that we warned the reader about our inability to present direct clinical implication of some observed results, e.g. the sixth paragraph of the Discussion section:

"It has to be noted, however, that the magnitude of cytotoxic effects determined by metal particles is significantly higher in in vitro conditions compared to tissues or living organisms, therefore our data do not have a direct clinical implication".

We want to clarify, once again, that all the potential clinical effects reported in the Discussion section are examples of the limitations that should be overcome if such protocols 
were brought to further stages in in vitro research, e.g. applications with organoids and immune cells. None of these "implications" were mentioned in the Results section.

- $\quad$ Regarding the fourth comment:

Firstly, as widely clarified in our previous reply, we have already meticulously reported the claimed information on page two of our manuscript:

“In 2006, Human Brain Wave (HBW) designed Rigenera ${ }^{\circledR}$, a chairside technology introduced as an innovative technique that allows isolation of stem cells from autologous micro-grafts for application in clinical regenerative procedures. Using the Rigenera ${ }^{\circledR}$ protocol, in just one surgical procedure, the patient is both donor and recipient of calibrated micro-grafts. This takes place within a single procedural time and is capable of enriching micro-graft derived progenitors cells for regeneration within the recipient site [7]."

Secondly, the aims of our study have already been highlighted in our reply to Comment 1.

In summary, we conducted our study as a novel pilot study to explore whether or not Rigenera protocol could be amenable in its current form for in vitro applications. We first undertook a "quality check" starting our sample processing from single cells suspension ("to characterize the effect of Rigenera ${ }^{\circledR}$ workflow on the viability of primary cultured fibroblasts and on the quality of the processed samples"), with the potential future aim to move forward towards two-dimensional cell layers, and 3D tissue samples, such as spheroids and organoids. Cell culture plates of primary fibroblasts isolated form the oral mucosa still contain an abundant progenitor cell population. This is demonstrated e.g. by effective and prompt osteogenic, adipogenic, and condrogenic induction if the cells are exposed and cultured in dedicated media. Unfortunately, our preliminary findings at the first step based on single cells prevented us from taking this protocol forward as the results were not suggestive of further potential to advance our hypothesis.

It is the opinion of these authors that the title of our article is not misleading at all.

Rigenera ${ }^{\circledR}$ has been defined as a "Progenitor Cell-Enriching Device".

These authors have simply used Rigenera's working function of the device in the title, instead of its commercial name. The reasons behind this choice are multiple. First of all, to avoid setting any precedent for any conflict of interest. Secondly, to avoid any sort of covert advertising in the title. Finally, for a scientific style issue, to make sure that appropriate mesh terms were used in the title for indexing purposes and therefore could be captured by scientists in the field.

To conclude, the direct paraphrase of the title is "Suitability of Rigenera for In Vitro Applications". These authors cannot see any other shorter/clearer titles for this article, and we strongly believe that there is no space in it for any deceit.

- Regarding the last comment, and the conclusions depicted by HBW representatives:

These authors would like to clarify, once again, that we pointed out some drawbacks in the use of the Rigenera ${ }^{\circledR}$ technology and protocol, specifically in the tested experimental conditions. We provided incontrovertible evidence that metal particles were produced even in the negative control, and we were able to characterise these. The experimental conditions in which these data were obtained are clearly reported. No mention to clinical data, e.g. biocompatibility data, has been made because this was completely out of scope from our study. Our study was not planned as a clinical study and has not assessed or judged any aspect of the clinical chair-side use of the device.

We agree with the company that some of the comparisons we made with some clinical scenarios could confound inattentive readers, and therefore were prompt to clarify in the Discussion section that "The presence of metal particles around implants has been reported in the literature for decades, and a recent systematic review highlighted that titanium particles surrounding peri-implant tissues are a common finding [24]." We are experienced academics and know very well that it is fully pertinent to discuss the implications of the results in the Discussion section of a scientific article. Please refer to "Reply to Comment 2" for further details on this aspect. 
We also did not question any bureaucratic and/or certification aspect of the device, having certainty that a device sold in over 40 countries clearly must have met high standards for clinical applications.

These authors find the words "confusing" and "impolite" extreme and inappropriate, particularly when applied to the scientific community, and we believe that they reflect a biased approach to our scientific findings. These authors are certain that the scientific community has the necessary skills to properly assess our article content, discriminating crucial pieces of information such as protocols and manufacturer instructions.

We can only be happy that Rigeneracons ${ }^{\circledR}$ has been approved by several regulatory agencies, and that the device has almost 15 years of preclinical data and clinical data gained. However, all of these points were not mentioned as they were completely out of the scope of our original article. We feel we have already reported a huge amount of information and references for the device. Surprisingly, the only point worth bringing forth to the discussion, namely the described discrepancy of the pore size of the Rigeneracons ${ }^{\circledR}$, has been cautiously and widely skipped by Astarita et al. in their letter.

These authors would also like to mention that we presented genuine data produced by world-class facilities and state-of-the-art equipment, such as the FEI Tecnai F30 scanning electron microscope (SEM), while analysing components of Rigeneracons ${ }^{\circledR}$ technology. In spite of the diameter of $50 \mu \mathrm{m}$ reported by the manufacturer, the pore diameter size was found to range between 250 and $400 \mu \mathrm{m}$, with an average of around $300 \mu \mathrm{m}$. What the manufacturer describes to the public is the diameter, and we have provided incontrovertible evidence that their description is inaccurate. This inconsistency is so straightforward that it does not need any further comment. We encourage HBW to be more critical with their own work, rather than arguing against clear and simple scientific evidence obtained by respected scientists.

Author Contributions: Conceptualization, A.C. and N.C.; original draft preparation, A.C. and N.C.; writing-review and editing, A.C., T.Y., G.P., R.P., M.M., N.C. All authors have read and agreed to the published version of the manuscript.

Funding: This research received no external funding.

Informed Consent Statement: Not applicable.

Data Availability Statement: Data sharing is not applicable to this article.

Conflicts of Interest: The authors declare no conflict of interest.

\section{References}

1. Astarita, C.; Trovato, L.; Graziano, A. Comment on Celentano et al. Suitability of a Progenitor Cell-Enriching Device for In Vitro Applications. Coatings 2021, 11, 146. Coatings 2021, 11, 636. [CrossRef]

2. Celentano, A.; Yap, T.; Pantaleo, G.; Paolini, R.; McCullough, M.; Cirillo, N. Suitability of a Progenitor Cell-Enriching Device for In Vitro Applications. Coatings 2021, 11, 146. [CrossRef]

3. Xie, Y.; Lampinen, M.; Takala, J.; Sikorski, V.; Soliymani, R.; Tarkia, M.; Lalowski, M.; Mervaala, E.; Kupari, M.; Zheng, Z.; et al. Epicardial transplantation of atrial appendage micrograft patch salvages myocardium after infarction. J. Heart Lung Transplant. 2020, 39, 707-718. [CrossRef] [PubMed]

4. Jimi, S.; Kimura, M.; De Francesco, F.; Riccio, M.; Hara, S.; Ohjimi, H. Acceleration Mechanisms of Skin Wound Healing by Autologous Micrograft in Mice. Int. J. Mol. Sci. 2017, 18, 1675. [CrossRef] [PubMed] 